\title{
Number and distribution of myofibroblasts and $\alpha$-smooth muscle actin expression levels in fetal membranes with and without gestational complications
}

\author{
XIAOYAN MA ${ }^{1,2}$, FAN YANG $^{2}$, SHULI YANG ${ }^{2}$, AZHAR RASUL $^{1,3}$, TING LI $^{3}$, \\ LIANLIAN LIU ${ }^{2}$, MIN KONG ${ }^{2}$, DONGMEI GUO ${ }^{2}$ and TONGHUI MA ${ }^{1,3}$ \\ ${ }^{1}$ Membrane Channel Research Laboratory, Northeast Normal University, Changchun, Jilin 130024; \\ ${ }^{2}$ Department of Obstetrics and Gynecology; ${ }^{3}$ Central Research Laboratory, Jilin University Bethune Second Hospital, \\ Changchun, Jilin 130041, P.R. China
}

Received February 7, 2014; Accepted November 7, 2014

DOI: $10.3892 / \mathrm{mmr} .2015 .3719$

\begin{abstract}
The permeability and tension of fetal membranes (FMs) is associated with extracellular matrix proteins produced largely by myofibroblasts (MFBBs). $\alpha$-smooth muscle actin $(\alpha-$ SMA) is involved in the contraction of MFBBs and has been implicated as a special biomarker of MFBBs in non-vessel FM. The present study demonstrated, by using immunohistochemistry, reverse-transcription polymerase chain reaction and western blotting, that MFBBs were mainly distributed in chorioamniotic mesoderm at 16-21 weeks and in chorionic mesoderm at 22-40 weeks, respectively, while overlapping with each other at 16-40 weeks. In addition, a quantity of MFBBs were identified in chorionic epithelia at 16-40 weeks. The MFBBs were distributed parallel to the FMs. The quantities of MFBBs and the expression levels of $\alpha$-SMA were negatively associated with increasing gestational progress and of amniotic fluid indexes in full-term females (those from oligohydramnios were higher than polyhydramnios); however, the thickness of the FM's mesoderm remained unchanged. Of note, the number of MFBBs in early-onset severe pre-eclampsia (EOSP) was significantly decreased in comparison with that in EOSP controls and late-onset severe pre-eclampsia (LOSP), while that in LOSP was higher than that in LOSP controls. The present data indicated that the changes in the quantity and distribution of MFBBs in the FM affects the permeability and tension of the FM. In addition, the findings suggested that the expression levels of $\alpha$-SMA in the FM also contributed to the properties of the FM. Simultaneously, the number and distribution of MFBBs and the expression levels
\end{abstract}

Correspondence to: Dr Tonghui Ma, Central Research Laboratory, Jilin University Bethune Second Hospital, 218 Ziqiang Street, Changchun, Jilin 130041, P.R. China

E-mail: tonghuima@jlu.edu.cn

Key words: myofibroblast, $\alpha$-smooth muscle actin, tension, fetal membranes, permeability, amniotic-fluid indexes, pre-eclampsia of $\alpha$-SMA in the FM may be involved in the mechanisms of development, apoptosis and trophoblast-MFBB transformation of the FM.

\section{Introduction}

The fetal membrane (FM) is a complex consisting of the amnion and the chorion, constituting the fetal components and the decidua, a maternal component. It surrounds the fetus and amniotic fluid throughout the gestational period and is critical in providing a protective screen for the fetus and in regulating the amniotic fluid volume $(1,2)$. Throughout pregnancy, the increasing area of the FM, the development and maintenance of permeability and tension of the FM are required to fulfill the demands for increasing fetal weight, fetal size and amniotic fluid volume (1). Evidence from human studies demonstrated the presence of five aquaporins (AQPs; AQP1, 3, 4, 8 and 9) and water transport channels in epithelial cells of the human FM. In addition, the intramembranous amniotic fluid regulation is controlled by alterations in AQP expression, which is associated with oligohydramnios and polyhydramnios (2). At present, it is accepted that extracellular matrix (ECM) proteins and a fibrous mesh structure from an assemblage of collagen fibers in the FM's mesoderm are major contributors to the permeability and the tension of the FM mesoderm $(3,4)$. The increased thickness and density of connective tissues, which predominantly consists of collagen components, have been implicated in the elevated tension of the FM but also reduce the permeability of the FM (3). Thus, the two contradictory properties of the FM were not completely explained by changes in thickness and density of the FM connective tissue $(3,4)$.

Fibroblasts and myofibroblasts (MFBB), two main cell components in the FM mesoderm $(5,6)$, possess a potential for producing collagen and other ECM proteins in connective tissues (6). In the FM mesoderm cells, the fibroblasts are relatively quiescent under normal conditions and function to maintain tissue homeostasis by regulating the turnover of the ECM. MFBBs are a more active cell type, producing more abundant collagen, other ECM proteins and matrix metalloproteinases (MMPs) and are thus important in tissue 
remodeling $(5,7)$. In addition to producing ECM proteins, the highly contractile properties of MFBBs have been noted (7). Wang and Schneider (6) suggested that the MFBB in the human amniotic membrane and the chorionic membrane may contribute to the protection of the FM from overdistension. Previous studies have elucidated that the differentiation and the increase in the numbers of MFBB in the FM may implicate FM rupture and premature labor (5).

$\alpha$-smooth muscle actin ( $\alpha$-SMA), a cytoskeletal protein component in MFBBs and smooth muscle cells, is perceived as a potential mechanosensitive protein $(8,9)$, closely associated with the contraction and the stretch effects of MFBBs and smooth muscle cells (8). However, MFBBs are hypothesized to have a hybrid phenotype between fibroblasts and smooth muscle cells. Therefore, it is difficult to identify MFBBs among the stromal cells based upon cell phenotype. Of note, a feature unique to free human chorioamniotic membranes is the absence of fetal vessels and other tissues containing smooth muscle cells during all stages of development (10). Thus, $\alpha$-SMA serves as a specific biomarker of MFBBs in FM mesoderm.

Phenotypic switching between fibroblasts and MFBBs is a common event (11). MFBBs are mainly derived from fibroblast differentiation under conditions of mechanical microenvironmental change (12), tissue injury (13) and hypoxia (11). In addition, MFBBs may be derived from macrophage transdifferentiation and epithelial-mesenchymal transition $(14,15)$. In addition, MFBBs may also dedifferentiate into fibroblasts as apoptosis occurs (15).

With the advance of gestation, the FM goes through stages of development and proliferation prior to reaching mature stages which occur simultaneously with apoptosis. Pre-eclampsia is a serious complication of gestation associated with placental hypoxia (16). Oligohydramnios and polyhydramnios are also gestational complications associated with abnormal amniotic fluid volume. However, the numbers and the distribution of MFBBs and $\alpha$-SMA expression in the FM in a normal pregnancy at differing weeks of gestation, in full-term pregnancy with variable amniotic fluid indexes (oligohydramnios, normal amniotic fluid volume and polyhydramnios) and in the two subtypes of severe pre-eclampsia, early onset severe pre-eclampsia (EOSP) and late-onset severe pre-eclampsia (LOSP) are largely unknown.

The present study assessed differences in the quantities and distribution of MFBBs and the expression levels of $\alpha$-SMA in the FM in normal pregnant females at different weeks of gestation. These differences were also assessed in patients with oligohydramnios and polyhydramnios as well as EOSP and LOSP. The present study also investigated the transformation of chorionic trophoblasts into MFBBs. The findings of the present study provided novel insight for improving the understanding of the mechanisms of FM development under the physiological conditions of normal gestation and in cases of gestational complication.

\section{Materials and methods}

Research subjects and specimen collection. The present study was approved by the ethics committee of Jilin
University Bethune Second Hospital (Changchun, China), and performed with informed maternal consent. Samples were obtained from the Department of Obstetrics, Jilin University Bethune Second Hospital. A total of 79 pregnant females hospitalized for delivery between January 2010 and March 2012 were selected as research subjects. Normal pregnancies $(n=30,16-40$ weeks) at different gestational weeks were divided into four groups according to gestational week: Early ( $\geq 16-<22$ weeks, $n=7)$, early/mid ( $\geq 22-<28$ weeks, $n=7)$, mid/late ( $\geq 28-<34$ weeks, $\mathrm{n}=8)$ and late $(\geq 34-\leq 40$ weeks, $n=8)$, respectively. Full-term pregnancies (37-40 weeks, n=30) were divided into three groups of 10 according to amniotic-fluid index: Oligohydramnios, (amniotic fluid indexes $<8$ ); normal (amniotic fluid indexes 8-18); polyhydramnios, (amniotic fluid indexes $>18$ ), including six cases of the previously mentioned full term pregnancies and four cases of other full-term pregnancies. Based on onset time of pre-eclampsia, patients $(n=25)$ with severe pre-eclampsia (SP; diastolic blood pressures $\geq 110 \mathrm{mmHg}$ and/or systolic blood pressures $\geq 160 \mathrm{mmHg}$ on at least three occasions, $>5 \mathrm{~g}$ protein per 24 in urine) were designated to the EOSP group ( $n=13, \geq 20-<34$ weeks) with an earlier onset of morbidity ( $\geq 14$ days) and LOSP $(n=12$; $\geq 34$ - $\leq 40$ weeks) with a later onset of morbidity ( $<5$ days). Subjects had no other obstetric or gynecological complications. No statistical differences were identified in the ages of the participants $(\mathrm{P}>0.05)$.

Following delivery of the placentas, three sections of FM $(3.0 \mathrm{~cm} \times 3.0 \mathrm{~cm})$ in the central and outer regions and were immediately biopsied under aseptic conditions. Simultaneously, decidual membranes from half of each section of FM were removed and subsequently stored at $-80^{\circ} \mathrm{C}$ prior to reverse transcription polymerase chain reaction (RT-PCR) and western blot analysis. Additional sections were fixed with $4 \%$ formaldehyde for $24 \mathrm{~h}$, embedded in paraffin and cut into $3-\mu \mathrm{m}$ sections, dried at $65^{\circ} \mathrm{C}$ for $7.5 \mathrm{~h}$ and subsequently stored at room temperature prior to immunohistochemical analysis.

Immunohistochemistry. The primary antibody, ( $\alpha$-SMA mouse monoclonal, cat. no. ZM-0003), secondary antibody kit [poly-horseradish peroxidase anti-mouse/rabbit immunoglobulin (Ig)G, PV-9000 2-step plus] and 3,3'-diaminobenzidine (DAB) kit were purchased from Zhongshan Goldenbridge Biotechnology Co., Ltd. (Beijing, China). Standard procedures were followed on the $3-\mu \mathrm{m}$ sections. Antigen retrieval was performed for $20 \mathrm{~min}$ at moderate temperature $\left(93-97^{\circ} \mathrm{C}\right)$ in a microwave. In order to quench the activity of endogenous peroxidase, slides were placed in $3 \%$ hydrogen peroxide at room temperature for $10 \mathrm{~min}$. The slides were subsequently incubated with primary antibody $(1 / 200)$ for $60 \mathrm{~min}$ at $37^{\circ} \mathrm{C}$ in a humidified chamber. Following this, secondary antibody was added to the slides for $50 \mathrm{~min}$ at $37^{\circ} \mathrm{C}$. Subsequently, the DAB kit was used to detect the staining of $\alpha$-SMA in the FM sections. The primary antibody was replaced with phosphate-buffered saline as a negative control. Placental vessels were used as a positive control. Slides were assessed by two independent pathologists in 10 random fields of vision for each slide (BX51; Olympus Corporation, Tokyo, Japan). 
Table I. Quantities of MFBBs in the FM and the thickness of FM mesoderm in normal pregnancies at different gestational weeks.

\begin{tabular}{lcccc}
\hline Group (n) & Age (years) & Gestation time (weeks) & MFBBs (n) & Thickness (cm) \\
\hline Early (7) & $29.7 \pm 4.9$ & $18.2 \pm 2.3$ & $55.1 \pm 41.0$ & $2.8 \pm 1.5$ \\
Early/mid (7) & $28.0 \pm 5.9$ & $24.2 \pm 2.4$ & $27.6 \pm 8.7$ & $2.1 \pm 0.6$ \\
Mid/late (8) & $32.0 \pm 3.4$ & $30.3 \pm 4.6$ & $21.2 \pm 7.6$ & $2.5 \pm 1.5$ \\
Late (8) & $28.0 \pm 3.6$ & $38.0 \pm 1.8$ & $18.4 \pm 3.6$ & $2.9 \pm 1.4$ \\
\hline
\end{tabular}

Values are presented as the mean \pm standard deviation. Early, $\geq 16$ weeks - $<22$ weeks; early/mid, $\geq 22$ weeks - $<28$ weeks; mid/late, $\geq 28$ weeks - $<34$ weeks; late, $\geq 34$ weeks - $\leq 40$ weeks. The quantities of FM mesoderm MFBBs: in the four groups, P=0.063; early vs. early-mid, $\mathrm{P}=0.129$; early vs. mid-late, $\mathrm{P}=0.072$; early vs. late, $\mathrm{P}=0.06$; early-mid vs. mid-late, $\mathrm{P}=0.155$; early-mid vs. late, $\mathrm{P}=0.047$; mid-late vs. late, $\mathrm{P}=0.516$. The thickness of FM mesoderm in the four groups, $\mathrm{P}=0.34$. FM, fetal membrane; MFBB, myofibroblast; thickness, the thickness of FM mesoderm.

$R T-P C R$. Total RNA was extracted from the fresh FM samples with TRIzol reagent (Invitrogen Life Technologies, Carlsbad, CA, USA) and cDNA was reverse transcribed using a Superscript First-strand Synthesis System (Invitrogen Life Technologies). The cDNA was used as a template for PCR amplification using the following primers: $\alpha-S M A$ forward, 5'-GCGTGGCTATTCCTTCGTTAC-3' and reverse, 5'-CATAGTGGTGCCCCCTGATAG-3' (331 bp); and GAPDH forward, 5'-GAAGGTGAAGGTCGGAGT-3' and reverse, 5'-GAAGATGGTGATGGGATTTC-3' (226 bp). The primers were designed using Primer Premier software, version 5.0 (Premier Biosoft, Palo Alto, CA, USA), and were synthesized by Sangon Biotech Co., Ltd. (Shanghai, China). GAPDH was used as an internal control. The amplicon size was 331 base pairs. The PCR products were analyzed using Image-Pro Plus 6.0 software (National Institutes of Health, Bethesda, MD, USA).

Immunoblotting. The FM specimens were homogenized in lysis buffer (50 mM Tris- $\mathrm{HCl}$ (pH 8.0), $150 \mathrm{mM} \mathrm{NaCl}$, $1 \%$ triton $\mathrm{X}-100,0.5 \%$ sodium deoxycholate, $1 \%$ NP-40 and $1 \% \mathrm{SDS}$ ) on ice and were centrifuged at 13,800 $\mathrm{x} \mathrm{g}$ for $8 \mathrm{~min}$ at $40^{\circ} \mathrm{C}$. The protein was separated by $10 \%$ SDS-PAGE and transferred onto a polyvinylidene difluoride (PVDF) membrane (Bio-Rad Laboratories, Inc., Hercules, CA, USA). Following blocking with $5 \%$ non-fat milk (w/v) and washing with $0.1 \%$ Tween 20 Tris-buffered saline, the PVDF membrane was incubated with primary $\alpha$-SMA mouse monoclonal antibody $(1: 1,000 ; \mathrm{ZM}-0003)$ overnight at $4^{\circ} \mathrm{C}$, washed and subsequently incubated with peroxidase conjugated affinipure goat anti-mouse IgG (1:1,000; ZB-2301; Zhongshan Goldenbridge Biotechnology Co, Ltd.) and detected using an enhanced chemiluminescence system (cat. no. WBKLS0100; EMD Millipore, Billerica, MA, USA).

Statistical analysis. The database was established using Excel 2003 (Microsoft, Redmond, WA, USA) and SPSS 17.0 (SPSS, Inc., Chicago, IL, USA) statistical software. The data met the criteria of a normal distribution and were presented as the mean \pm standard deviation. Comparisons between groups were performed using one-way analysis of variance. A $\chi^{2}$ test was used to establish whether the prevalence among the groups was significantly different. $\mathrm{P}<0.05$ was considered to indicate a statistically significant difference.

\section{Results}

Distribution of MFBBs and the thickness of mesoderm in FMs. In the present study, it was reported for the first time, to the best of our knowledge, that the majority of MFBBs in FM mesoderm were interweaved with each other in pregnant females with and without gestational complications. MFBBs and fibroblasts were generally distributed parallel to the FM (Fig. 1A a-1-4, Fig. 2A a-1-3 and Fig. 3A a-1-4). Immunohistochemical results demonstrated that MFBBs were mainly distributed in chorioamniotic mesoderm at 16-21 weeks and in chorionic mesoderm at 22-40 weeks. Furthermore, it was observed that the fibroblasts were predominantly positioned in the amniotic mesoderm at 22-40 weeks (Fig. 1A a-1-4). Of note, MFBBs stained with antibodies against $\alpha$-SMA were noted among chorionic epithelial cells with and without gestational complications (Fig. 1A a-1-4, Fig. 2A a-1-3 and Fig. 3A a-1-4). No significant differences $(\mathrm{P}=0.34 ; \mathrm{P}=0.53 ; \mathrm{P}=0.60)$ were identified in the thicknesses of the FM mesoderm in normal pregnancies at different gestational weeks, in full-term pregnancies with different amniotic fluid indexes and in females with SP irrespective of onset and morbidity time (Tables I-III).

Quantity of MFBBs and the expression levels of $\alpha$-SMA in the $F M$. In the present study, although notable differences in the quantities of MFBBs were identified in 10 random visual fields in the same FM slides with and without gestational complications, correlations of MFBB-quantity changes were observed in the FM. In normal pregnancies at different gestational weeks (early, $\geq 16$ weeks - $<22$ weeks; early/mid, $\geq 22$ weeks - $<28$ weeks; $\mathrm{mid} /$ late, $\geq 28$ weeks - $<34$ weeks; late, $\geq 34$ weeks - $\leq 40$ weeks), the quantity of MFBBs in FM mesoderm decreased with advancement of gestation and were negatively correlated with gestational weeks ( $r=-0.47, \mathrm{P}=0.008$; Fig. $1 \mathrm{~A}$ a-1-4, A b and A c). Variable quantities of MFBBs were present; however, differences were not statistically significant $(\mathrm{P}=0.063)$ in the four groups at different gestational weeks (Fig. 1A a-1-4 and Table I). The results demonstrated that with the increase in the number of gestational weeks, the expression levels of $\alpha$-SMA mRNA in the FM were decreased in normal pregnancies (Fig. 1B a and $\mathrm{B}$ b). Similarly to the expression levels of $\alpha$-SMA mRNA in FM, the expression levels of $\alpha$-SMA protein in FM also significantly and gradually decreased with progression of gestational weeks in normal pregnancies (Fig. $1 \mathrm{C}$ a and $\mathrm{C} \mathrm{b}$ ). 
Table II. Quantity of MFBBs in the FM and the thickness of FM mesoderm in full term pregnancies with different amniotic fluid indexes.

\begin{tabular}{lcccc}
\hline Group (n) & Age (years) & $\begin{array}{c}\text { Gestation time } \\
\text { (weeks) }\end{array}$ & $\begin{array}{c}\text { Amniotic-fluid } \\
\text { indexes }\end{array}$ & $\begin{array}{c}\text { Thickness } \\
(\mathrm{cm})\end{array}$ \\
\hline Oligohydramnios (10) & $29.5 \pm 4.5$ & $39.0 \pm 0.8$ & $6.0 \pm 1.4$ & $33.1 \pm 21.6$ \\
Normal (10) & $29.2 \pm 5.6$ & $39.0 \pm 1.1$ & $13.5 \pm 2.2$ & $21.3 \pm 5.3$ \\
Polyhydramnios (10) & $28.1 \pm 3.4$ & $39.0 \pm 0.3$ & $21.7 \pm 3.6$ & $12.4 \pm 4.0$ \\
\hline
\end{tabular}

Data are presented as the mean \pm standard deviation. Oligohydramnios, amniotic fluid indexes <8; normal, amniotic fluid indexes 8-18; polyhydramnios, $>18$. Number of FM-mesoderm MFBBs: oligohydramnios vs. normal, $\mathrm{P}=0.137$; polyhydramnios vs. normal, $\mathrm{P}=0.001$; polyhydramnios vs. oligohydramnios, $\mathrm{P}=0.015$. Thickness of $\mathrm{FM}$ mesoderm in the three groups, $\mathrm{P}=0.53$. FM, fetal membrane; MFBB, myofibroblast.

Table III. Quantities of MFBBs in the FM and the thickness of FM mesoderm in females with SP.

\begin{tabular}{|c|c|c|c|c|}
\hline Group (n) & Age (years) & $\begin{array}{c}\text { Gestation time } \\
\text { (weeks) }\end{array}$ & MFBBs (n) & $\begin{array}{c}\text { Thickness } \\
(\mathrm{cm})\end{array}$ \\
\hline EOSP (13) & $27.2 \pm 2.7$ & $26.8 \pm 4.2$ & $12.9 \pm 4.0$ & $2.3 \pm 0.7$ \\
\hline EOSP Control (13) & $28.8 \pm 2.1$ & $25.7 \pm 3.5$ & $42.6 \pm 32.8$ & $2.8 \pm 1.1$ \\
\hline LOSP (12) & $28.3 \pm 3.2$ & $37.9 \pm 1.8$ & $37.3 \pm 23.5$ & $2.4 \pm 0.3$ \\
\hline LOSP Control (12) & $29.2 \pm 4.0$ & $37.8 \pm 1.6$ & $17.8 \pm 23.5$ & $2.4 \pm 0.8$ \\
\hline
\end{tabular}

Data are presented as the mean \pm standard deviation. Quantities of FM-mesoderm MFBBs: EOSP vs. EOSP control, P=0.000; EOSP vs. LOSP, $\mathrm{P}=0.004$; LOSP vs. LOSP control, $\mathrm{P}=0.015$. Thickness of FM-mesoderm in the four groups, $\mathrm{P}=0.60$. FM, fetal membrane; MFBB, myofibroblast; SP, severe pre-eclampsia; EOSP, early onset SP; LOSP, late onset SP.

In full-term pregnancies with different amniotic-fluid indexes (oligohydramnios, amniotic-fluid indexes $<8$; normal amniotic-fluid indexes, amniotic-fluid indexes 8-18; polyhydramnios, amniotic-fluid indexes $>18$ ), the quantity of MFBBs in FM mesoderm decreased $(\mathrm{r}=-0.66, \mathrm{P}<0.001)$ with elevation of amniotic fluid indexes. Significant differences $(\mathrm{P}=0.001)$ were identified in the cell quantities of MFBBs in the three groups with different amniotic-fluid indexes (Fig. 2A a-1-4, $\mathrm{A} \mathrm{b}$ and A c; Table II). A decrease in the expression levels of $\alpha$-SMA mRNA and $\alpha$-SMA protein in the FM along with elevation of amniotic-fluid indexes was observed in the three groups (Fig. 2B a and B b; Fig. 2C a and $\mathrm{C} \mathrm{b}$ ).

Cell quantities of MFBBs in FM mesoderm were decreased in patients with EOSP compared with those in normal controls at matched gestational weeks $(\mathrm{P}<0.001)$ and those in LOSP patients. By contrast, LOSP patients exhibited an increase $(\mathrm{P}=0.004)$ in MFBB cell quantities in the FM mesoderm in comparison to those in normal controls at matched gestational stages $(\mathrm{P}=0.015$; Fig. $3 \mathrm{~A}$ a-1-4 and $\mathrm{A}$ b; Table III).

\section{Discussion}

The present study revealed that MFBBs, which are capable of generating collagen protein, other ECM proteins (8) and MMPs $(9,17)$ are the principal cell components of the FM mesoderm (amniotic mesoderm and chorionic mesoderm) at 16-22 weeks of gestation. These findings suggested that MFBBs are critical in regulating the increased rate of development of FM mesoderm at the earlier phases of gestation. Between 22-40 weeks, the later and slower developmental phases of FM mesoderm, the relatively quiescent fibroblasts were the principal cell components in amniotic mesoderm. This indicated that fibroblasts are the main contributors in regulating the basic turnover of the ECM and the tensile force of the amniotic mesoderm, functioning to maintain amniotic homeostasis. Simultaneously, the more active MFBBs were present in chorionic mesoderm, suggesting that the increase in permeability and tensile force of the FM mesoderm may be associated with the functions of MFBBs in the chorionic mesoderm. In addition, no significant differences were identified in the thickness of the FM mesoderm in normal pregnancies at different gestational stages, between the patients with different amniotic fluid indexes and the patients with EOSP and LOSP. Thus, the slight alterations in FM mesoderm thickness may not be an important factor in the changes in permeability and tensile force of the FM along with gestational progression. As the cell quantities of MFBBs in the FM mesoderm and the expression levels of $\alpha$-SMA mRNA and $\alpha$-SMA protein in the FM decreased with progressive gestational weeks, it was hypothesized that the changes in MFBB quantities and $\alpha$-SMA expression levels were significant factors in regulating the permeability and the tensile force of the FM mesoderm. Fibroblasts and MFBBs, the latter in particular, are considered to be capable of producing actin-myosin interaction-mediated cell traction forces (CTFs) $(18,19)$, which are relatively slow, sustained and non-reversible compared with 

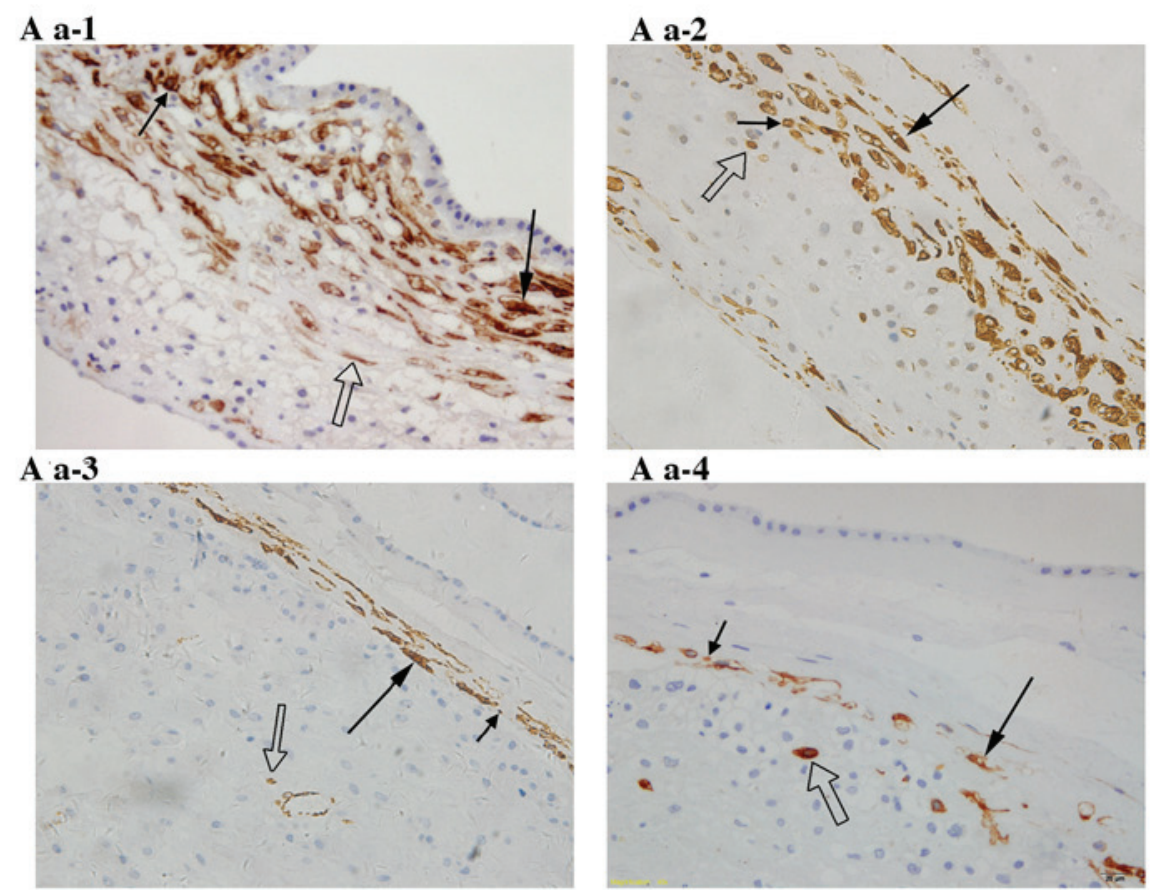

A b

A c
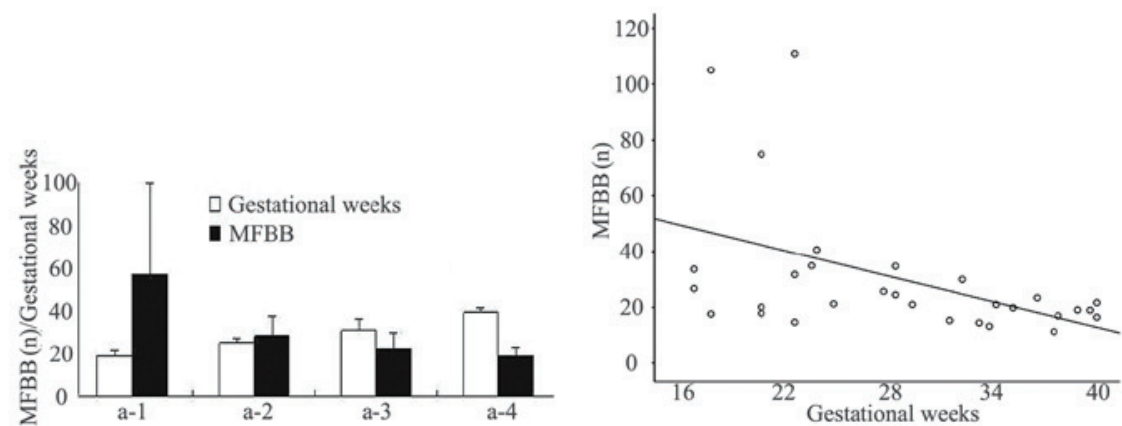

B a

\section{B b}
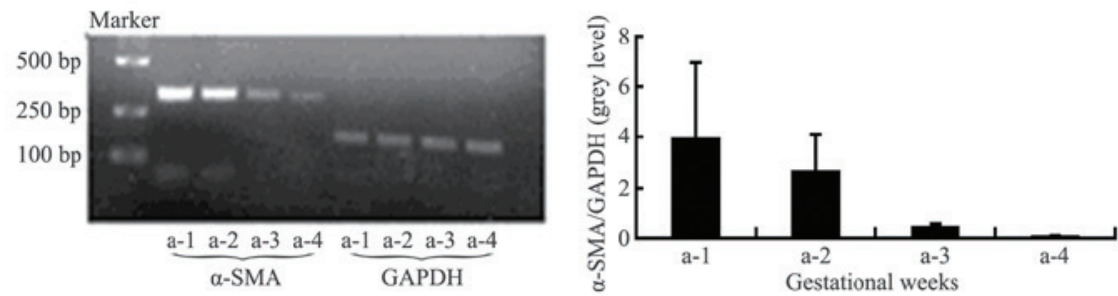

$\mathbf{C} \mathbf{a}$

C b
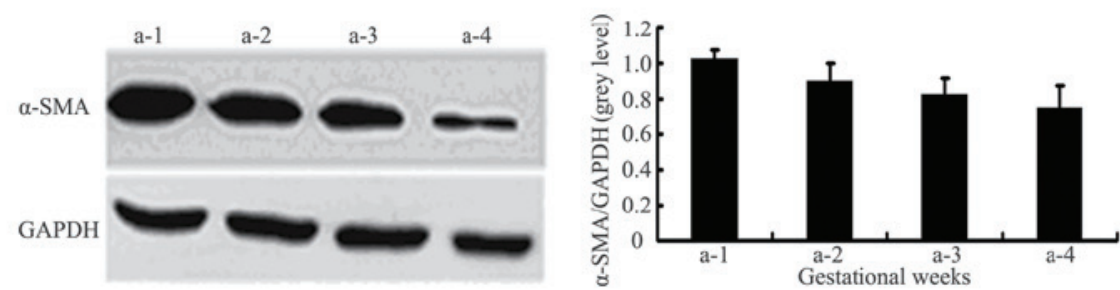

Figure 1. Number and distribution of MFBBs and $\alpha$-SMA expression levels in FMs at different gestational weeks. (A a-1) Early: $\geq 16$ weeks $-<22$ weeks. (A a-2) Early/mid: $\geq 22$ weeks - $<28$ weeks. (A a-3) Mid/late: $\geq 28$ weeks - $<34$ weeks. (A a-4) Late: $\geq 34$ weeks - $\leq 40$ weeks. (Magnification, x200). Long arrows indicate MFBBs with vertical section; short arrows indicate MFBBs with transverse section; hollow arrows indicate MFBBs in chorionic epithelia. The majority of MFBBs in FM mesoderm was distributed parallel to FM and interweaved with each other and separated by the ECM. Fibroblasts were mostly positioned in the amniotic mesoderm at 22 weeks - 40 weeks. There were differences among the MFBB numbers in the four groups, but they were not statistically significant ( $\mathrm{P}=0.063$ ). (A b, A c) The numbers of MFBBs were descending along with elevation of gestational weeks. (B a, C a) Expression of $\alpha$-SMA mRNA was detected at $331 \mathrm{bp}$ as a single band, GAPDH served as internal control and the expression of $\alpha$-SMA mRNA and $\alpha$-SMA protein became weaker with advance of gestation weeks. (B b, C b) $\alpha$-SMA mRNA band grey values/GAPDH mRNA band grey values and $\alpha$-SMA protein band grey values/GAPDH protein band grey values were decreasing with advance of gestation weeks. FM, fetal membrane; MFBB, myofibroblast; SP, severe pre-eclampsia; EOSP, early onset SP; LOSP, late onset SP; $\alpha$-SMA, $\alpha$-smooth muscle actin; ECM, extracellular matrix. 

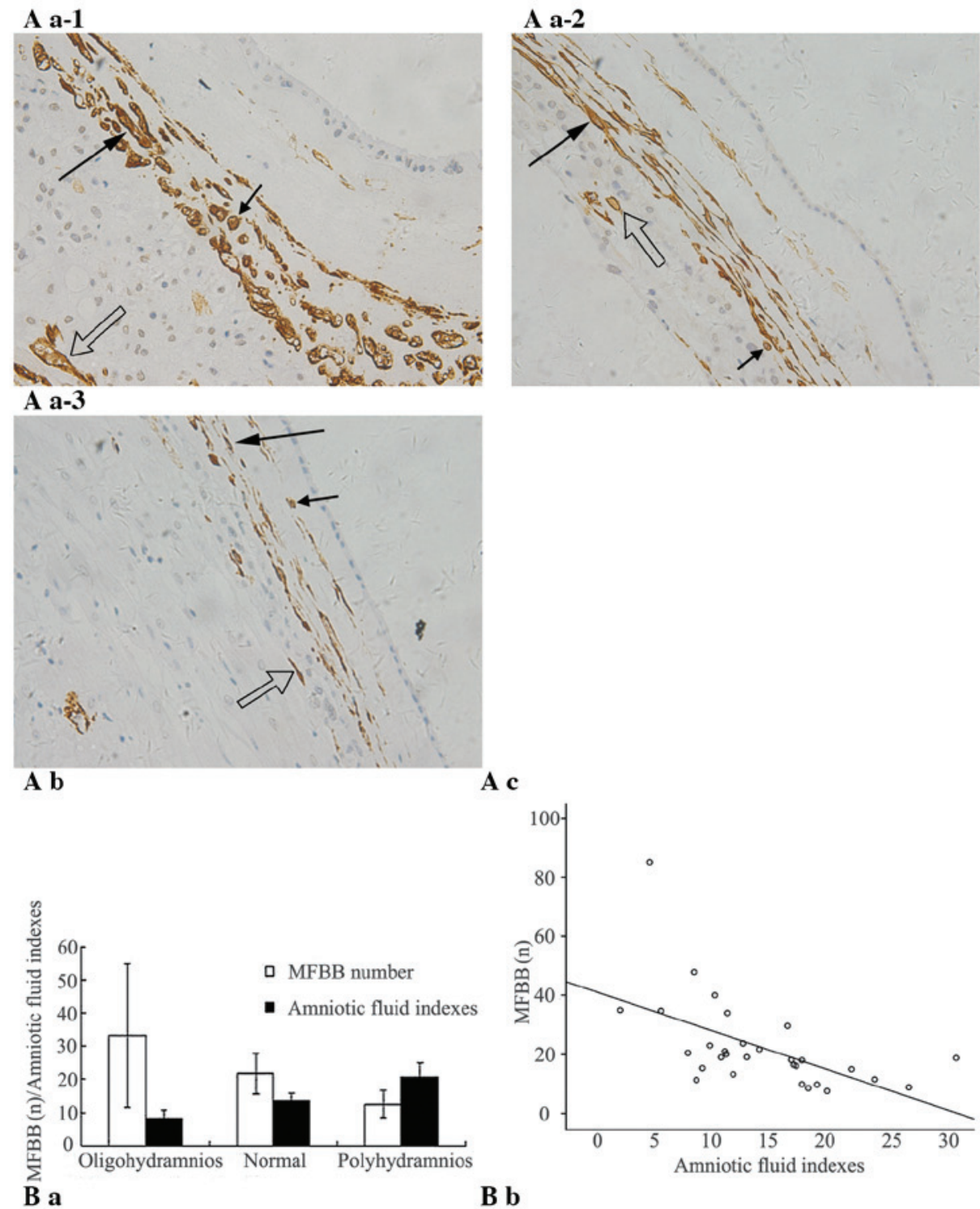

B a

\section{B b}
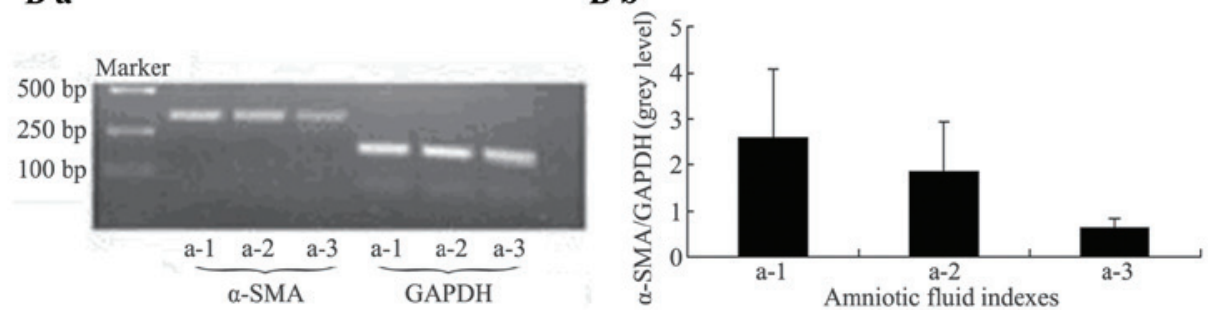

$\mathrm{C}$ a

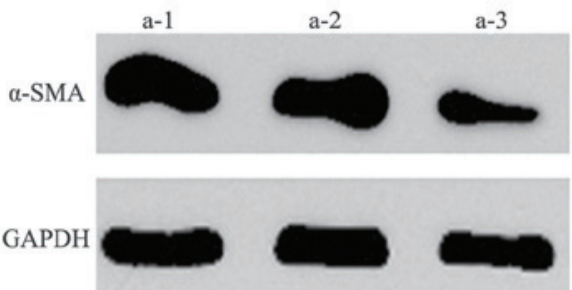

$\mathbf{C} \mathbf{b}$

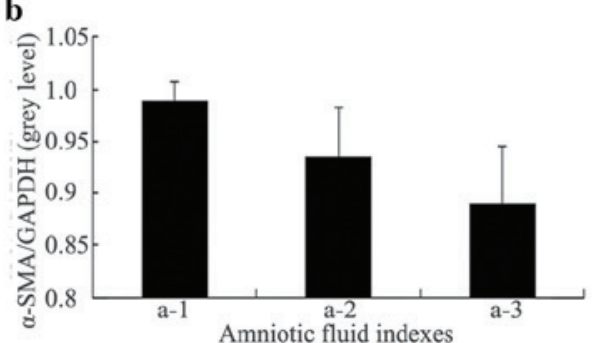

Figure 2. Number and distribution of MFBBs and $\alpha$-SMA expression levels in full term FMs with different amniotic fluid indexes. (A a-1) Oligohydramnios, amniotic fluid indexes <8. (A a-2) Normal, amniotic fluid indexes 8-18. (A a-3) Polyhydramnios, amniotic fluid indexes $>18$. Long arrows indicate MFBBs with vertical section; short arrows indicate MFBBs with transverse section; hollow arrows indicate MFBBs in chorionic epithelia. The majority of MFBBs in FM mesoderm was distributed parallel to FM and interweaved with each other and separated by the ECM. There were significant differences ( $\mathrm{P}=0.001)$ among the MFBB numbers in the three groups (magnification, x200). (A b, A c) The number of MFBBs was descending along with increase of amniotic fluid indexes. (B a, C a) Expression of $\alpha$-SMA mRNA and $\alpha$-SMA protein became weaker with increase of amniotic-fluid indexes. (B b, C b) $\alpha$-SMA mRNA band grey values/GAPDH mRNA band grey values and $\alpha$-SMA protein band grey values/GAPDH protein band grey values were decreasing with advance of gestation weeks. FM, fetal membrane; MFBB: myofibroblast; SP, severe pre-eclampsia; EOSP: early onset SP; LOSP: late onset SP; $\alpha$-SMA, $\alpha$-smooth muscle actin; ECM, extracellular matrix. 
A a-1

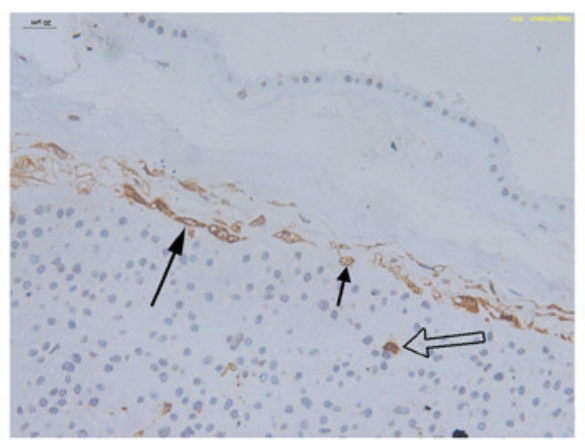

A a-3

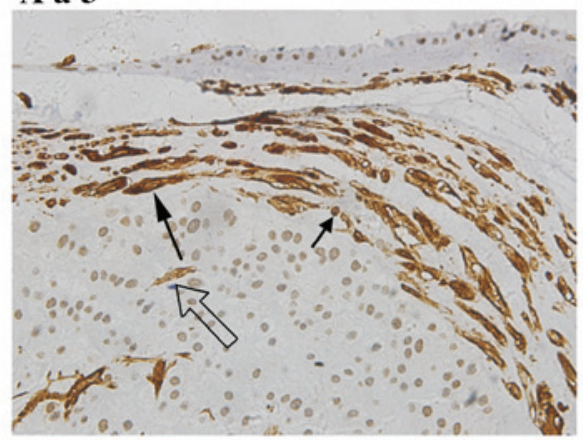

A b

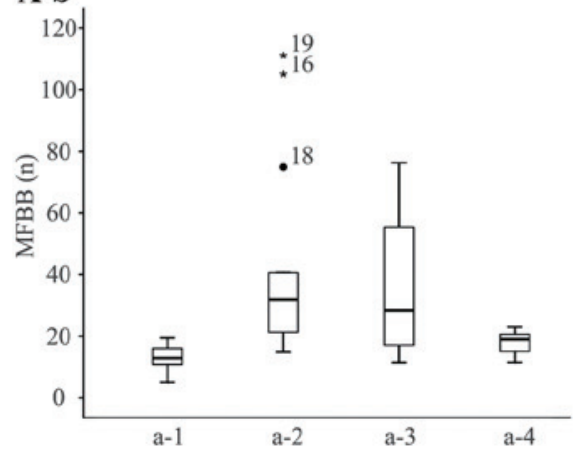

\section{A a-2}

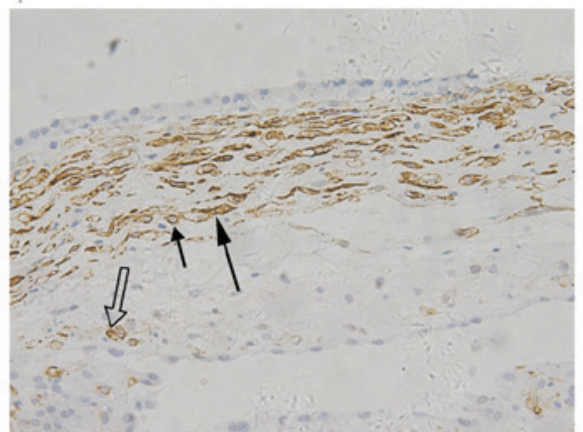

A a-4

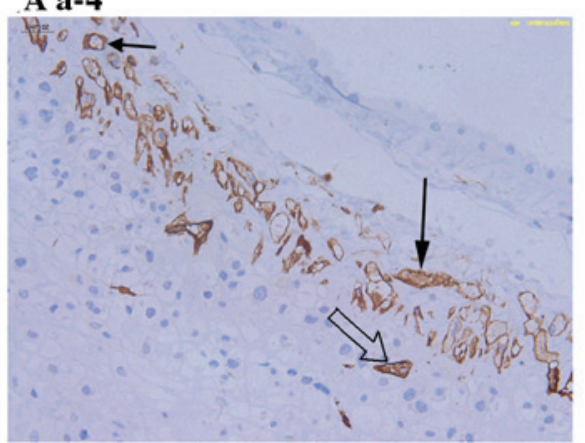

Figure 3. Number of FM-mesoderm MFBBs in EOSP and LOSP. (A a-1) EOSP. (A a-2) EOSP control. (A a-3) LOSP. (A a-4) LOSP control. (Magnification, $\mathrm{x} 200)$. Long arrows indicate MFBBs with vertical section; short arrows indicate MFBBs with transverse section; hollow arrows indicate MFBBs in chorionic epithelia. The MFBBs were distributed parallel to $\mathrm{FM}$ and interweaved with each other and separated by the $\mathrm{ECM}$. $\mathrm{A}$ significant decrease $(\mathrm{P}<0.001 ; \mathrm{P}=0.004)$ of MFBB numbers was observed in EOSP compared to EOSP controls and LOSP, whereas a significant increase (P=0.015) in MFBB numbers was identified in LOSP compared to LOSP controls. (A b) Comparison among the numbers of MFBBs in EOSP, EOSP control, LOSP and LOSP control. FM, fetal membrane; MFBB, myofibroblast; SP, severe pre-eclampsia; EOSP, early onset SP; LOSP, late onset SP; $\alpha$-SMA, $\alpha$-smooth muscle actin; SP, severe pre-eclampsia; EOSP, early onset SP; LOSP, late onset SP.

the $\mathrm{Ca}^{2+}$-regulated rapid and reversible contraction of smooth muscle cells $(20,21)$. The formation process of CTFs includes the following route: Intracellular tension by fibroblasts and MFBBs is generated through adenosine-triphosphate-powered sliding of actin-myosin filaments and transfer of this tension to the ECM via local adhesion to both ends of the stress fiber $(22,23)$. In addition to producing interaction-mediated cell traction, MFBBs also generate contractile forces through contraction of $\alpha$-SMA, a type of cytoskeletal protein of MFBBs that is a potential mechanosensitive protein $(8,9)$ and involved in the contraction of collagen protein and other ECM proteins $(24,25)$. The present study also demonstrated that the MFBBs and the fibroblasts in the FM mesoderm were distributed parallel to the FM and the MFBBs were interweaved with each other. The distribution characteristics of MFBBs and fibroblasts in the FM mesoderm may be beneficial to tensile force, intracellular tension and contraction. These observations may partially explain the direct or indirect roles of MFBBs in regulating the permeability, interaction-mediated cell traction and contraction of FM mesoderm.

Previous studies demonstrated that no AQP and no water transport channels are present in FM mesoderm, while FM permeability has been established to increase with gestational progression as well as the tensile force of the FM. However, the permeability and the tensile force of FM, a pair of conflicting FM properties, have been hypothesized to be dependent on the turnover of collagen protein and ECM proteins. The present study demonstrated that in full-term pregnancies, no significant changes were present in the thickness of FM mesoderm accompanied by polyhydramnios, oligoamnios and normal volume amniotic fluid. Finally, these findings endorsed the basic function of MFBBs and fibroblasts in the maintenance 
of connective tissue thickness of FM mesoderm in order to co-ordinate these conflicting FM properties. Simultaneously, the numbers of MFBBs in FM mesoderm and the expression trends of $\alpha$-SMA mRNA and $\alpha$-SMA protein in the FM were significantly and negatively correlated $(r=-0.66, P<0.001)$ with amniotic fluid indexes. The greater quantities of MFBBs and the higher expression levels of $\alpha$-SMA mRNA and $\alpha$-SMA protein were present in the FM accompanied by oligoamnios, whereas the FM accompanied by polyhydramnios was characterized by a lesser quantity of MFBBs and lower expression levels of $\alpha$-SMA mRNA and protein. These results indicated that the quantity differences and distribution states of MFBBs in FM mesoderm and the expression levels of $\alpha$-SMA in the FM may be important in affecting the permeability of FM mesoderm, inducing changes to amniotic fluid volume. There are a number of conflicting studies regarding the effect of hypoxia on MFBB cell numbers and $\alpha$-SMA expression levels. Rogers et al (26) demonstrated that the structure of intercellular actin, a cytoskeletal protein, is disrupted under hypoxic conditions. A study by Modarressi et al (27) revealed that hypoxia impairs the differentiation and function of skin MFBB, elucidated through quantifying $\alpha$-SMA expression and cell contraction in collagen gels and on wrinkling silicone substrates. Misra et al (11) reported that hypoxia induces a phenotypic switch of fibroblasts to MFBBs via an MMP-2/tissue inhibitors of metalloproteinase-mediated pathway. Pre-eclampsia is a common and serious complication of gestation involving hypoxia. Based on the onset time of pre-eclampsia, it may be grouped as early-onset pre-eclampsia (onset time, $<34$ weeks) and late-onset pre-eclampsia (onset time, $>34$ weeks). Depending on the severity of pre-eclampsia, the disorder is also classified into mild pre-eclampsia and severe pre-eclampsia (28). Generally, early onset pre-eclampsia is the more severe subtype of pre-eclampsia. Emerging evidence from animal studies, including, human trials, has implicated that placental ischemia and hypoxia may be a central causative factor in the onset and development of pre-eclampsia (29). Of note, in the present study, significant differences were noted among the MFBB numbers in FM mesoderm between EOSP and EOSP controls with matched gestational stages and between LOSP and LOSP controls with matched gestational stages as well as between EOSP and LOSP. The quantity of MFBBs in FM mesoderm from EOSP patients with a longer time period of morbidity was significantly lower than that in EOSP controls and LOSP patients with a shorter time period of morbidity. Furthermore, the number of MFBBs in FM mesoderm from LOSP patients was significantly higher than that in the LOSP controls. The results indicated that a different onset time and different duration of hypoxia may lead to the variable effects of MFBBs on proliferation or apoptosis and the expression levels of $\alpha$-SMA in MFBBs.

Chorionic trophoblasts are considered to be well-differentiated epithelial cells; however, their function remains to be elucidated, while it is likely that they act as a protective barrier. In the present study, it was noted that there were a number of MFBBs labeled with $\alpha$-SMA antibody in the trophoblast layers of chorionic membranes from normal pregnancies and those with gestational complications. This was consistent with previous studies $(14,15)$, suggesting that certain epithelial cells may differentiate into mesenchymal cells. The epithelial cells of certain types of cancer can transform into mesenchymal cells of the cancer tissue and human retinal pigment epithelial cells may transform into cells similar to MFBBs (30). The results of the present study suggested that chorionic trophoblasts have the potential to differentiate into MFBBs.

In conclusion, the present study revealed that the differences in quantity and the distribution states of MFBBs in FM mesoderm as well as the $\alpha$-SMA expression levels in the FM may be the main contributors to the permeability, tensile force and intracellular tension of the FM by affecting the turnover of collagen protein and other ECM proteins and also the contractility, proliferation and apoptosis of MFBBs. In addition, the onset time and persistence of hypoxia in MFBBs may induce differential impacts on MFBB numbers and $\alpha$-SMA expression levels. Ultimately, chorionic trophoblasts may have the potential to differentiate into MFBBs.

\section{Acknowledgements}

The authors would like to thank Professors Hongwen Gao and Mei Sun (Department of Pathology, Jilin University Bethune Second Hospital, Changchun, China) for helpful discussions during the study of histology and immunohistochemistry in the placental and fetal membrane samples. This study was supported by the Science and Technology Department of Jilin Province, China (grant no. 20090464) and the Science and Technology Agency of Changchun, China (grant no. 08SF44).

\section{References}

1. Minh HN, Douvin D, Smadja A and Orcel L: Fetal membrane morphology and circulation of the liquor amnii. Eur J Obstet Gynecol Reprod Biol 10: 213-223, 1980.

2. Sha XY, Xiong ZF, Liu HS, Di XD and Ma TH: Maternal-fetal fluid balance and aquaporins: from molecule to physiology. Acta Pharmacol Sin 32: 716-720, 2011.

3. Fujisato T, Tomihata K, Tabata Y, Iwamoto Y, Burczak K and Ikada Y: Cross-linking of amniotic membranes. J Biomater Sci Polym Ed 10: 1171-1181, 1999.

4. Jabareen M, Mallik AS, Bilic G, Zisch AH and Mazza E: Relation between mechanical properties and microstructure of human fetal membranes: an attempt towards a quantitative analysis. Eur J Obstet Gynecol Reprod Biol 144 Suppl 1: S134-S141, 2009.

5. McParland PC, Taylor DJ and Bell SC: Myofibroblast differentiation in the connective tissues of the amnion and chorion of term human fetal membranes-implications for fetal membrane rupture and labour. Placenta 21: 44-53, 2000.

6. Wang T and Schneider J: Fine structure of human chorionic membrane. Ultrastructural and histochemical examinations. Arch Gynecol 233: 187-198, 1983.

7. Kruidenier L, MacDonald TT, Collins JE, Pender SL and Sanderson IR: Myofibroblast matrix metalloproteinases activate the neutrophil chemoattractant CXCL7 from intestinal epithelial cells. Gastroenterology 130: 127-136, 2006.

8. Li B and Wang JH: Fibroblasts and myofibroblasts in wound healing: force generation and measurement. J Tissue Viability 20: 108-120, 2011.

9. Powell DW, Mifflin RC, Valentich JD, Crowe SE, Saada JI and West AB: Myofibroblasts. I. Paracrine cells important in health and disease. Am J Physiol 277: C1-C9, 1999.

10. Hoyes AD: Ultrastructure of the mesenchymal layers of the human chorion laeve. J Anat 109: 17-30, 1971.

11. Misra S, Fu AA, Misra KD, Shergill UM, Leof EB and Mukhopadhyay D: Hypoxia-induced phenotypic switch of fibroblasts to myofibroblasts through a matrix metalloproteinase 2/tissue inhibitor of metalloproteinase-mediated pathway: implications for venous neointimal hyperplasia in hemodialysis access. J Vasc Interv Radiol 21: 896-902, 2010. 
12. Szczodry M, Zhang J, Lim C, Davitt HL, Yeager T, Fu FH and Wang JH: Treadmill running exercise results in the presence of numerous myofibroblasts in mouse patellar tendons. J Orthop Res 27: 1373-1378, 2009.

13. Ross R, Everett NB and Tyler R: Wound healing and collagen formation. VI. The origin of the wound fibroblast studied in parabiosis. J Cell Biol 44: 645-654, 1970.

14. Yang J and Liu Y: Dissection of key events in tubular epithelial to myofibroblast transition and its implications in renal interstitial fibrosis. Am J Pathol 159: 1465-1475, 2001.

15. Carlson MA, Longaker MT and Thompson JS: Wound splinting regulates granulation tissue survival. J Surg Res 110: 304-309, 2003

16. Sankaralingam S, Arenas IA, Lalu MM and Davidge ST: Preeclampsia: current understanding of the molecular basis of vascular dysfunction. Expert Rev Mol Med 8: 1-20, 2006.

17. Tomasek JJ, Gabbiani G, Hinz B, Chaponnier C and Brown RA: Myofibroblasts and mechano-regulation of connective tissue remodelling. Nat Rev Mol Cell Biol 3: 349-363, 2002.

18. Hinz B, Phan SH, Thannickal VJ, Galli A, Bochaton-Piallat ML and Gabbiani G: The myofibroblast: one function, multiple origins. Am J Pathol 170: 1807-1816, 2007.

19. Sanger JW, Sanger JM and Jockusch BM: Differences in the stress fibers between fibroblasts and epithelial cells. J Cell Biol 96: 961-969, 1983.

20. Hinz B and Gabbiani G: Mechanisms of force generation and transmission by myofibroblasts. Curr Opin Biotechnol 14: 538-546, 2003.

21. Katoh K, Kano Y, Amano M, Onishi H, Kaibuchi K and Fujiwara K: Rho-kinase - mediated contraction of isolated stress fibers. J Cell Biol153: 569-584, 2001.

22. Balaban NQ, Schwarz US, Riveline D, Goichberg P, Tzur G, Sabanay I et al: Force and focal adhesion assembly: a close relationship studied using elastic micropatterned substrates. Nat Cell Biol 3: 466-472, 2001
23. van Beurden HE, Von den Hoff JW, Torensma R, Maltha JC and Kuijpers-Jagtman AM: Myofibroblasts in palatal wound healing: prospects for the reduction of wound contraction after cleft palate repair. J Dent Res 84: 871-880, 2005.

24. Bogatkevich GS, Tourkina E, Abrams CS, Harley RA, Silver RM and Ludwicka-Bradley A: Contractile activity and smooth muscle alpha-actin organization in thrombin-induced human lung myofibroblasts. Am J Physiol 285: L334-L343, 2003.

25. Liu T and Hu XD: Transdifferentiation of fibroblasts into myofibroblasts in the skin lesion of systemic sclerosis: role of transforming growth factor betal and its signal transduction. Nan Fang Yi Ke Da Xue Xue Bao 31: 1840-1845, 2011, (In Chinese).

26. Rogers KR, Morris CJ and Blake DR: The cytoskeleton and its importance as a mediator of inflammation. Ann Rheum Dis 51: 565-571, 1992.

27. Modarressi A, Pietramaggiori G, Godbout C, Vigato E, Pittet B and Hinz B: Hypoxia impairs skin myofibroblast differentiation and function. J Invest Dermatol 130: 2818-2827, 2010.

28. von Dadelszen P, Magee LA and Roberts JM: Subclassification of preeclampsia. Hypertens Pregnancy 22: 143-148, 2003.

29. George EM and Granger JP: Recent insights into the pathophysiology of preeclampsia. Expert Rev Obstet Gynecol 5: 557-566, 2010.

30. Ma J, Zhang Q, Moe MC and Zhu T: Regulation of cell-mediated collagen gel contraction in human retinal pigment epithelium cells by vascular endothelial growth factor compared with transforming growth factor-beta. Clin Experiment Ophthalmol 40: e76-e86, 2012. 